\title{
Variations
}

Variations

Revue internationale de théorie critique

$19 \mid 2016$

Critique des humanités numériques

\section{Présentation du dossier}

Les sciences humaines et sociales au prisme du digital turn

Fabien Granjon

\section{OpenEdition}

Journals

Édition électronique

URL : http://journals.openedition.org/variations/726

DOI : $10.4000 /$ variations.726

ISSN : 1968-3960

Éditeur

Les amis de Variations

Référence électronique

Fabien Granjon, «Présentation du dossier », Variations [En ligne], 19 | 2016, mis en ligne le 06 avril 2016, consulté le 30 avril 2019. URL : http://journals.openedition.org/variations/726 ; DOI : 10.4000/ variations.726

Ce document a été généré automatiquement le 30 avril 2019.

Les ami•e•s de Variations 


\title{
Présentation du dossier
}

\author{
Les sciences humaines et sociales au prisme du digital turn
}

\author{
Fabien Granjon
}

1 Depuis une dizaine d'années, on rencontre, d'abord au sein d'une littérature très spécialisée, puis de plus en plus dans les productions des sciences humaines et sociales prenant pour objet les technologies numériques d'information et de communication, le syntagme " humanités numériques » (digital humanities). L'expression semble faire suite à la mention "humanities computing", laquelle désignait un sous-champ des humanités utilisant des ressources informatiques et dont les travaux de Roberto Busa (1980) sur Saint Thomas d'Aquin (Index Thomisticus) avaient marqué la naissance (Burnard, 2012; Hockey, 2004). À partir du mitan des années 2000, la dénomination «humanités numériques »(HN) a habillé la création de départements d'enseignement et de recherche (l'UCL Centre for Digital Humanities en Grande-Bretagne, le Laboratoire de cultures et humanités digitales en Suisse, etc.), d'institutions fédératrices (office of Digital Humanities, Alliance of Digital Humanities Organizations, etc.), de rencontres régionales, nationales et internationales (THATCamps, HASTAC, etc.), de catégories académiques, de revues ( Computers and the Humanities, puis Journal of Digital Humanities, Digital Humanities Quarterly, Digital Studies/Le champ numérique, etc.), d'archives, d'outils de collaboration, de projets éditoriaux, de traités, de readers, de manifestes, etc. En France, le vocable «humanités numériques» est notamment une mention ministérielle servant à identifier des formations universitaires, des laboratoires d'excellence, de nouvelles filières, etc. Depuis la rentrée 2014, on évoque également la possible création d'une filière "humanités numériques » au baccalauréat général et l'expression est maintenant largement rentrée dans le vocabulaire des acteurs de la représentation politique, à l'instar du Président François Hollande qui, dans un récent discours à l'occasion de l'inauguration de nouveaux locaux pour l'École des Chartes (9 octobre 2015), suggérait que «l'effort pour les humanités numériques soit non seulement maintenu, mais renforcé dans les années qui viennent ».

2 Dans le champ scientifique, depuis son utilisation séminale par Susan Schreibman, Ray Siemens et John Unsworth (2004), l'expression s'est assez largement diffusée tout en 
continuant à relever d'une appellation assez peu contrôlée (Alvaro, 2012). Il est aujourd'hui difficile de rendre compte avec précision quelles réalités sociales sont désignées par cette locution. Label peu stabilisé, il entend décrire, chez les «DHers» (i.e. les universitaires se réclamant des Digital Humanities) les plus "pragmatiques", l'utilisation accrue des technologies numériques au sein des humanités et, plus largement, des sciences humaines et sociales (SHS) ${ }^{1}$, suivant ainsi le mouvement des objets mêmes de ces disciplines, irrémédiablement de plus en plus numériques (Burnard, 2012). Chez les plus ambitieux d'entre eux (Berry, 2015), la mention recouvre plutôt l'émergence d'un supposé nouveau paradigme, au sens de Thomas Kuhn (2008); pour certains autres, elle relève davantage de la "next big thing", nouveau grand récit accompagnant la libéralisation du secteur de l'enseignement supérieur (Grusin, 2013). De fait, le numérique est à considérer à la fois comme lignée technique qui se trouve au principe d'un répertoire toujours plus large d'artefacts qui colonisent les différentes sphères sociales et les existences pour le meilleur et pour le pire, mais aussi comme une culture « dans le sens où elle met en place un nouveau contexte à l'échelle mondiale " (Doueihi, 2013: 33). Ce dernier terme tend à désigner, d'une part, une forme de littéracie particulière, c'est-àdire une acculturation et un intérêt (technique, éthique, politique, etc.) aux technologies numériques, ainsi qu'à la manière dont celles-ci renouvellent le continuum innovationdiffusion-appropriation-subjectivation des contenus culturels ; d'autre part, que le code n'est pas neutre et qu'il «véhicule des présupposés et des préjugés et qui, dans son déploiement, concrétise des imaginaires" (Doueihi, 2013: 13). Enfin, le vocable « culture » décrit un infléchissement, par le numérique, des conduites, des manières de faire, de penser ou de partager, des valeurs, des normes, etc., ainsi que de leurs éventuelles inscriptions institutionnelles (politiques, juridiques, etc.). La portée du numérique se distribuerait alors du simple déplacement de quelques habitudes pratiques (e.g. lecture sur écran, rapport à l'écrit) à des mutations d'ordre anthropologique touchant notamment aux « couplages cerveaux-technologies intellectuelles ", c'est-à-dire aux entrelacements des mémoires internes/externes (Noyer, 2010 - modalités de travail, de faire lien, de subjectivation, de cognition, effets environnementaux, etc.). À l'instar de Milad Doueihi (2011), on va ainsi jusqu'à évoquer la construction d'un «humanisme numérique " (Parry, 2012, Moatti, Bachelet, 2012) considérant le numérique comme un «nouveau processus civilisateur» (Doueihi, 2008) lançant aux humanités le défi de " comprendre et d'évaluer jusqu'à quel point les logiciels sont en passe de devenir notre culture, en même temps que la culture se trouve en voie rapide de logicialisation » (Berry, 2015 : xx). Faut-il alors considérer que les HN peuvent constituer une "nouvelle" anthropologie focalisée sur les sociétés contemporaines fortement technologisées et dont l'objectif est d'opérer une synthèse des sciences humaines, sociales et « exactes » à l'ère numérique? La chose serait alors conduite en prenant pour objet le «tournant numérique » dans ses diverses dimensions, mais également en s'appuyant sur ses potentialités instrumentales afin de produire, traiter et/ou diffuser les savoirs portant précisément sur cette "conversion numérique » (digital turn). Certains des plus fervents zélateurs des $\mathrm{HN}$ imaginent effectivement qu'elles pourraient jouer ce rôle de nouvelle science synthétique (Alvaro, 2012 ; Passeron, 1991), visant à explorer à nouveaux frais, via d'innovants instruments, une "nouvelle rationalité » touchant tous les domaines de l'homme et l'humanité, et ce, sous les conditions ouvertes par la numérisation du signe, la calculabilité, ainsi que les formes sémiotiques qui leur sont liées; à moins que les HN ne soient déjà obsolètes, au point de devoir être remplacées par des social data sciences 
(Boullier, 2015). De l'extension du domaine de la surenchère en milieu académique concurrentiel...

3 L'ensemble des contributions réunies dans ce dossier ont vocation à envisager les $\mathrm{HN}$ et, plus largement, certains aspects afférant au mouvement de numérisation du champ scientifique, à l'aune d'une perspective critique. Fabien Granjon et Christophe Magis interrogent ainsi «la prétention des humanités numériques à redistribuer les cartes des SHS » et reviennent sur l'ambition programmatique «révolutionnaire » que leur attribuent certains de leurs imprudents zélateurs. Les auteurs avancent que, malgré ces arrogances, les HN posent un ensemble de questions entrant en résonance avec certaines des exigences du principium de la critique (interdisciplinarité, totalisation, praxis, appropriation) et envisagent la nécessité du développement d'humanités numériques critiques. Dans un second article, ils discutent des nouvelles formes de mise à disposition du savoir scientifique (Open Access) qui sont généralement attenantes au développement des HN, mais trouvent leur source, plus lointainement, dans la concentration continue de l'édition scientifique. Magis et Granjon estiment que les réflexions sur l'accès libre auraient tout intérêt à prendre en considération les problématiques de base d'une économie politique critique visant plus largement à s'outiller pour un travail du négatif ayant comme horizon d'attente une « libération du travail scientifique ». En complément de ces deux premiers articles, Sébastien Broca propose de poser les bases d'une réflexion sur ce que l'on pourrait qualifier d'«idéologie du code», laquelle accompagne le développement d'une part non négligeable des digital humanities pour qui la logiciarisation du monde est souvent à la fois un objet et une méthode. Depuis un socle théorique puisé dans la pensée de Cornelius Castoriadis, le sociologue entend rappeler que l'on ne peut dissocier les choix épistémologiques des moyens grâce auxquels la connaissance est produite, et que les usages des technologies informatiques dans le cadre du champ scientifique embarquent nécessairement (et souvent implicitement) une conception du savoir dont il semble indispensable de discuter des fondements normatifs. Cette réflexion sur l'épistémologie du code est poursuivie par Arthur Lefebvre dont la contribution prend pour objet un «sous-champ » des HN, la simulation sociale, dont les attendus tiennent à la formalisation informatique des processus sociaux. Il interroge sous différents aspects " cette méthode d'expérience de pensée assistée par informatique, où des agents "autonomes, hétérogènes, et à la rationalité limitée en interactions locales dans un espace défini" reproduisent des phénomènes dynamiques dits "bottom-up" ". Lefebvre montre notamment la manière dont la simulation sociale envisage la production de connaissance et souligne certains de ses impensés. Le dossier se clôt sur la proposition de trois collègues historiens (Thomas Bouchet, Guillaume Carnino et François Jarrige) qui resituent le développement des HN dans un mouvement plus vaste, celui de la "numérisation de l'université ", lequel porte des enjeux d'ordre politique de toute première importance, liés au travail des divers personnels de l'Alma mater, aux modes de production de la science et à l'utilité des savoirs académiques, que le régime de production néolibéral des sciences n'oriente pas vraiment vers des fins premières émancipatoires.

4 À l'heure où le numérique est présenté par la sphère économico-politique comme le secteur qui permettra de relancer la croissance et la compétitivité de la France, le champ 
scientifique, non sans lien avec ce champ du pouvoir, est en train de faire des humanités numériques la nouvelle panacée de la production académique et, plus prosaïquement, le nouveau sésame permettant de recevoir des financements tant publics que privés. Force est de constater que la région épistémologique censée constituer le soubassement des HN reste pourtant des plus floues. Il n'y a, à ce jour, aucun accord franc sur un répertoire de nécessités, d'attendus, de méthodes ou de concepts qui permettrait de délinéer relativement clairement un espace interdisciplinaire singulier. Pour autant, une certaine collusio dans l'illusio, c'est-à-dire " une complicité foncière dans le fantasme collectif " (Bourdieu, 2004 : 19) de l'existence d'une «communauté scientifique $\mathrm{HN}$ » semble bel et bien prendre corps. La fortune présumée d'un «consortium HN» pousse d'ailleurs certains à essayer de faire coïncider celui-ci avec le périmètre de leur propre discipline et, ainsi, à préempter le domaine à leur profit, espérant sans doute trouver, par cette entremise, un surcroît de légitimité (Domenget et al., 2016). La " communauté HN » est donc, aujourd'hui, surtout constituée du rassemblement des prétendants à un renouvellement des rapports de pouvoir académiques; non, semble-t-il, pour en démonter les incuries et en réparer les injustices, mais plutôt pour rejouer, à nouveaux frais et à l'occasion du digital turn, un combat ayant pour enjeu l'instauration d'un nouveau monopole de la représentation scientifiquement légitime du réel et une nouvelle norme de la valeur scientifique, combat qui, espèrent-ils, leur apporteront les places auxquelles ils aspirent.

\section{BIBLIOGRAPHIE}

Alvaro (Rafael C.), « The Digital Humanities Situation », in Gold (Matthew K.) ed., Debates in the Digital Humanities, Minneapolis, University of Minnesota Press, 2012, pp. 50-55.

Berry (David M.), Critical Theory and the Digital, New York, Bloomsbury, 2015.

Boullier (Dominique), «Les sciences sociales ne jouent plus leur rôle de contre-pouvoir ", Le Monde, septembre 2015, p. 6.

Bourdieu (Pierre), Esquisse pour une autoanalyse, Paris, Raisons d'agir, 2004.

Burnard (Lou), « Du literary and linguistic computing aux digital humanities : retour sur 40 ans de relations entre sciences humaines et informatique », in Mounier (Pierre) dir., Read/Write Book 2. Une introduction aux humanités numériques, Marseille, OpenEdition Press, 2012, pp. 45-58.

Busa (Roberto), «The Annals of Humanities Computing: the Index thomisticus ", Computers and the Humanities, n 14, 1980, pp. 83-90, http://www.alice.id.tue.nl/references/busa-1980.pdf.

Domenget (Jean-Claude), Bonaccorsi (Julia), Carayol (Valérie), « Introduction au dossier

"Humanités numériques" et SIC », Revue française des sciences de l'information et de la communication , $\mathrm{n}^{\circ} 8,2016$, https://rfsic.revues.org/1984.

Doueihi (Milad), Qu'est-ce que le numérique ?, Paris, PUF, 2013.

Doueihi (Milad), Pour un humanisme numérique, Paris, Seuil, 2011. 
Doueihi (Milad), La grande conversion numérique, Paris, Seuil, 2008.

Grusin (Richard), « The Dark Side of the Digital Humanities - Part 2 », Thinking C21, 2013, http:// www.c21uwm.com/2013/01/09/the-dark-side-of-the-digital-humanities-part-2/.

Hockey (Susan), « The History of Humanities Computing », in Schreibman (Susan), Siemens (Ray), Unsworth (John) dir., A companion to Digital Humanities, Oxford, Blackwell Publishing, 2004, pp. 3-19.

Kuhn (Thomas S.), La structure des révolutions scientifiques, Paris, Champs sciences, 2008.

Moatti (Alexandre), Bachelet (Rémi), « Wikipédia, un projet hors-normes ? ", Annales des Mines, $\mathrm{n}$ ${ }^{\circ}$ 67, 2012, pp. 48-53.

Noyer (Jean-Max), « Connaissance, pensée, réseaux à l'heure numérique. Pour une nouvelle Renaissance ",

Les Cahiers du numérique, vol. 6, $\mathrm{n}^{\circ}$ 3, 2010, pp. 187-209.

Parry (Dave), « The Digital Humanities or a Digital Humanism », in Gold (Matthew K.) ed., Debates in the Digital Humanities, Minneapolis, University of Minnesota Press, 2012, pp. 429-437.

Passeron (Jean-Claude), Le raisonnement sociologique. L'espace non-poppérien du raisonnement naturel, Paris, Nathan, 1991.

Schreibman (Susan), Siemens (Ray), Unsworth (John), dir., A companion to Digital Humanities, Oxford, Blackwell Publishing, 2004.

\section{NOTES DE FIN}

1. Dans la sphère anglo-saxonne, les humanities n'ont pas de périmètre bien défini. Aussi, les sciences sociales sont-elles souvent incluses dans le label. Les digital humanities n'échappent pas à la règle.

INDEX

Mots-clés : champ scientifique, critique, digital humanities, humanisme numérique, humanités numériques

\section{AUTEUR}

\section{FABIEN GRANJON}

Professeur en Sciences de l'information et de la communication, Université Paris 8 - Cemti 